\title{
Effects of ECG Signal Processing on the Inverse Problem of Electrocardiography
}

\author{
Laura R Bear ${ }^{1}$, Y Serinagaoglu Dogrusoz ${ }^{2}$, J Svehlikova ${ }^{3}$, J Coll-Font ${ }^{4}$, W Good ${ }^{5}$, E van Dam ${ }^{6}$,

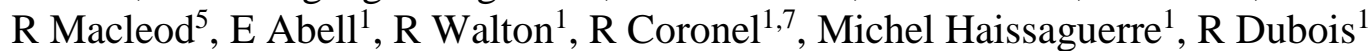 \\ ${ }^{1}$ IHU-LIRYC, Université de Bordeaux, Bordeaux, France. \\ ${ }^{2}$ Electrical and Electronics Engineering Department, Middle East Technical University, Turkey \\ ${ }^{3}$ Institute of Measurement Science, Slovak Academy of Sciences, Bratislava, Slovakia \\ ${ }^{4}$ Computational Radiology Department at Boston Children's Hospital, Boston (MA), USA \\ ${ }^{5}$ Dept. of Bioengineering and SCI Institute, University of Utah, Salt Lake City (UT), USA \\ ${ }^{6}$ Peacs BV, Nieuwerbrug aan den Rijn, The Netherlands \\ ${ }^{7}$ Dept. Exp. Cardiology, Academic Medical Center, Amsterdam, The Netherlands
}

\begin{abstract}
The inverse problem of electrocardiography is illposed. Errors in the model such as signal noise can impact the accuracy of reconstructed cardiac electrical activity. It is currently not known how sensitive the inverse problem is to signal processing techniques.

To evaluate this, experimental data from a Langendorffperfused pig heart $(n=1)$ suspended in a human-shaped torso-tank was used. Different signal processing methods were applied to torso potentials recorded from 128 electrodes embedded in the tank surface. Processing methods were divided into three categories i) highfrequency noise removal ii) baseline drift removal and iii) signal averaging, culminating in $n=72$ different signal sets. For each signal set, the inverse problem was solved and reconstructed signals were compared to those directly recorded by the sock around the heart.

ECG signal processing methods had a dramatic effect on reconstruction accuracy. In particular, removal of baseline drift significantly impacts the magnitude of reconstructed electrograms, while the presence of highfrequency noise impacts the activation time derived from these signals $(p<0.05)$.
\end{abstract}

\section{Introduction}

The inverse problem of electrocardiography (ECGI) reconstructs the electrical activity of the heart from densely sampled body-surface potentials and a patient-specific heart-torso geometry. This is a promising tool that is increasingly being used to guide ablation therapy of cardiac arrhythmias, and to help understand the mechanisms underlying various cardiac electrical disorders [1]. Despite the increase in clinical adoption of this technique, previous validation has demonstrated spatio-temporal variability in recovered electrograms, potentially constraining the accuracy with which arrhythmogenic substrates can be identified using this approach [2].

This inverse problem is ill-posed and errors such as noise in the torso potentials significantly impact the accuracy of reconstructed cardiac activity [3]. While signal processing can be used to remove high frequency noise and baseline drift from body surface potentials, it is unclear how effective the different methods are in improving ECGI reconstructions. Furthermore, it is unknown how ECGI reconstructions are affected by signal noise.

To help answer these questions, an international workgroup of researchers was formed at the Computers in Cardiology Conference 2017. The objective of this study is to present the initial results of the workgroup, evaluating the impact of different signal processing methods on a standard formulation of the inverse problem using a torso tank experimental model.

\section{Experimental Data}

The experimental protocol used to obtain this data has previously been described [4] and is summarized below. All experimental procedures were approved by the Directive 2010/63/EU of the European Parliament on the protection of animals used for scientific purposes and the local ethical committee.

Experimental data were obtained from an ex-vivo pig heart perfused in Langendorff mode, covered by a sock (108 electrodes) and suspended into a human-shaped torso tank with 128 electrodes embedded. Ventricular and tank potentials were recorded simultaneously (BioSemi, the 
Netherlands) at $2 \mathrm{kHz}$ for approximately $30 \mathrm{~s}$ during RV pacing. Afterwards, 3D rotational fluoroscopy (Artis, Siemens) was used to obtain the position of the epicardium and electrodes with respect to the torso tank.

\section{Signal Processing Methods}

Different signal processing methods were applied to the raw tank electrode potentials using a tree-like structure with three distinct stages (Figure 1). The first stage (FiltHigh) used methods to remove high-frequency noise. In the second stage, baseline drift removal (BDR) methods were applied. In the final stage, signal averaging (SA) was used by temporally aligning the pacing spikes of each beat. For the following analysis, signals were created by using different combinations of the filters of each stages, including the original raw potentials, culminating in a total of 72 different processed signal sets. SA was always used in conjunction with BDR. Precise details of all the methods can be found in [5].

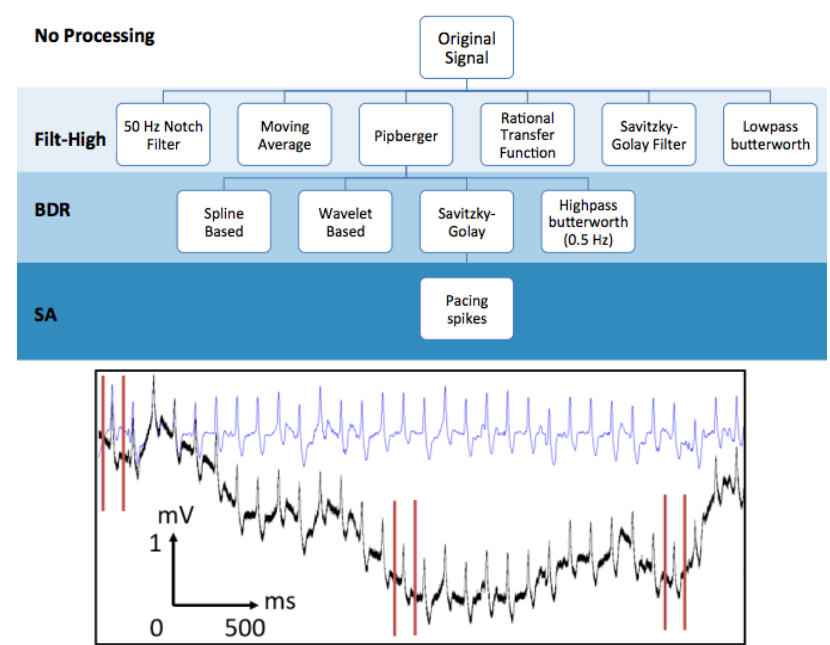

Figure 1. Tree-like structure (top) used to apply processing methods to the raw tank signals with example (bottom) of raw (black) and processed (blue) using a moving average and spline BDR filters (without SA). Three beats were selected for analysis (red bars).

\section{Inverse and Comparison Methods}

The boundary element method was used to define the forward matrix employing a homogeneous conductivity between the epicardial mesh (1012 points) and tank surface electrodes (128 electrode points). The inverse problem was solved using the Per C. Hansen's regularization toolbox in Matlab, using the Tikhonov zero-order regularization method[6]. The L-curve method [7] was used to define the regularization parameter $(\lambda)$.

For each signal set, the inverse problem was solved for the same 3 beats selected from the beginning, middle and end of the experiment (illustrated in Figure 1, red bars). As those with SA produced a single beat, reconstructed electrograms were compared to the un-averaged 3 beats recorded by the sock. Comparisons were made using Pearson's correlation and the root mean square (RMS) voltage. Activation times were defined by fitting a global activation field to activation delays between electrograms [8]. Activation was also compared using Pearson's correlation.

Differences were evaluated using a one-way ANOVA with statistical significance defined for $\mathrm{p}>0.05$. Results are presented as the median and range unless otherwise defined.

\section{Results}

The amplitude of reconstructed and recorded electrograms were compared using the RMS voltage, with examples plotted in Figure 2 (top). Electrograms reconstructed after BDR or SA (with or without Filt-High processing) had a mean RMS voltage up to four-fold larger than without any signal processing $(* \mathrm{p}<0.05)$, although they were still 0.7 to $1.2 \mathrm{mV}$ smaller than those recorded $(* * \mathrm{p}<0.0001)$. Combining the Pipberger filter with a high pass filter of $0.5 \mathrm{~Hz}$ and $\mathrm{SA}$ produced the largest reconstructed mean RMS voltages, although they were only $0.02 \mathrm{mV}$ larger than the next best methods.
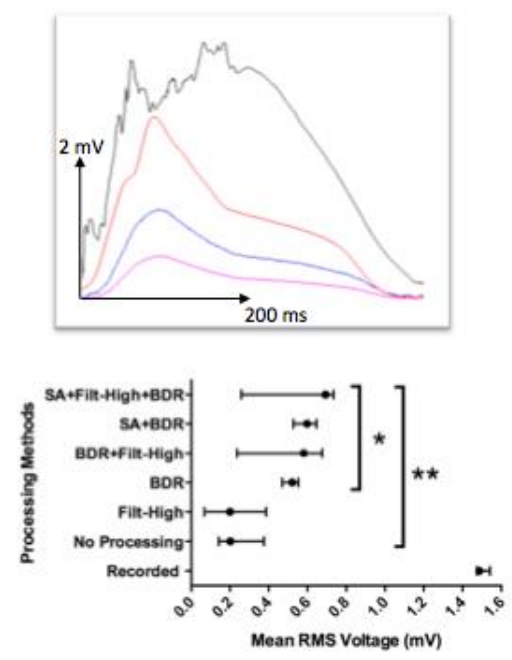

Figure 2 (top) RMS voltage from recorded (black) and reconstructed electrograms using torso signals without processing (blue), SA (red), and the Rational Transfer Function (purple). (Bottom) comparison of recorded and reconstructed mean RMS voltage using the different processing methods.

Figure 3 (top) presents examples of recorded (black) and reconstructed electrograms. Reconstructions with FiltHigh processing (purple) qualitatively showed smoother signals than without processing (blue). Reconstructed electrograms after BDR (red) were substantially noisier. 

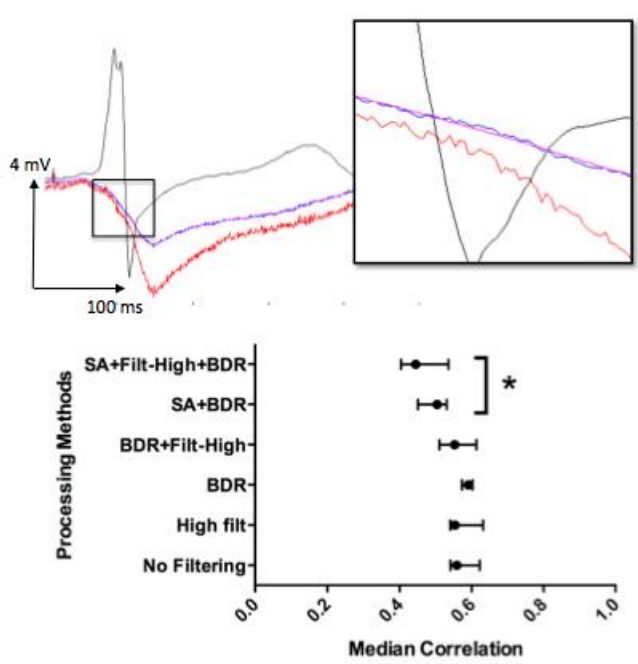

Figure 3 (top) Recorded (black) and reconstructed electrograms using no processing (blue), Savitzky-Golay BDR (red), and a $30 \mathrm{~Hz}$ low pas filter (purple). (Bottom) comparison of median correlation using the different processing methods.
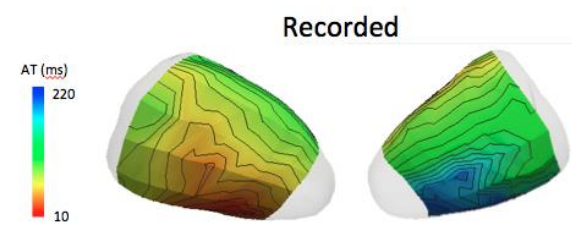

Reconstructed
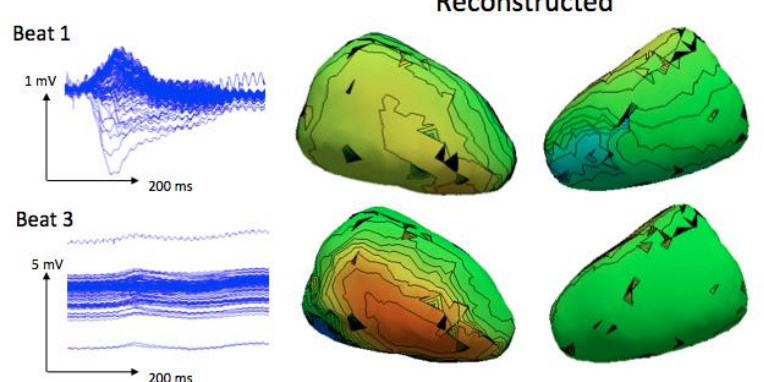

Figure 4. Torso signals (left) and activation maps (right) derived from recorded (top) and reconstructed electrograms using torso signals without processing for the first (middle) and third (bottom) selected beats.

Comparison of the median correlation between recorded and reconstructed electrograms calculated over the QRS complex showed there was no significant difference in correlation values between Filt-High and BDR compared to no processing ( $p>0.05)$. SA (with or without Filt-High) significantly reduced the median correlation with values 0.03 to 0.17 than those without processing $(\mathrm{p}<0.001)$. The highest correlation values were produced when a $60 \mathrm{~Hz}$ low pass filter was applied with BDR using a wavelet approach, with values 0.02 greater than without signal processing.

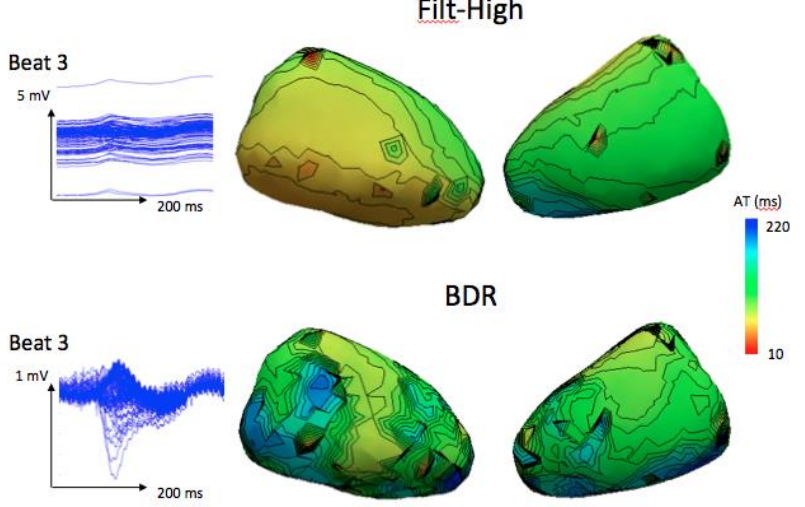

Figure 5. Torso signals (left) and activation maps (right) derived from reconstructed electrograms using $30 \mathrm{~Hz}$ lowpass filter (top) and a spline-based BDR (bottom).

Figure 4 and 5 present torso potentials (left) and activation maps (right) derived from recorded and reconstructed electrograms. In Figure 4 activation maps are shown for unprocessed signals for 2 different beats in the cycle. While the overall activation pattern is the same, there are substantial differences in timing between the two activation maps. In Figure 5, we see that torso potentials with Filt-High processing (but substantial baseline drift) reproduced accurate activation maps, while those from torso potentials with BDR and large amounts of high frequency noise are not.

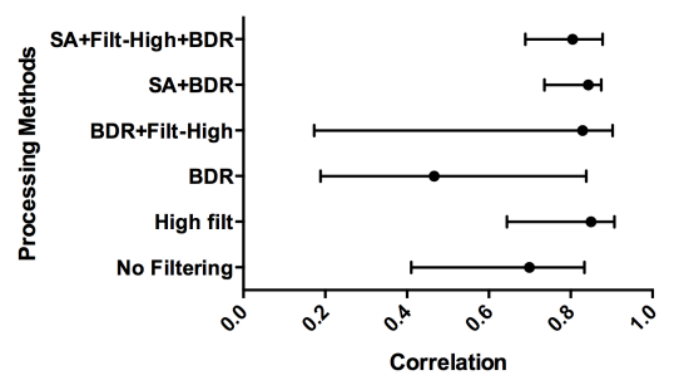

Figure 6. Correlation of recorded and reconstructed activation maps using the different processing methods.

While not significantly different ( $p>0.05$ ), Filt-High and SA substantially improved activation detection with higher median correlation values $(0.70$ vs 0.85 and 0.84 respectively) and a reduced range of correlation values from 0.42 to 0.27 and 0.13 respectively (Figure 6). All BDR methods except the wavelet based approached reduced accuracy of activation maps.

Figure 7 presents the $\lambda$ value used in the inverse reconstruction for each of the processed signals. Torso signals with BDR or SA (with or without Filt-High) resulted in significantly smaller $\lambda$ values than those without any processing or Filt-High alone $(\mathrm{p}<0.001)$. 


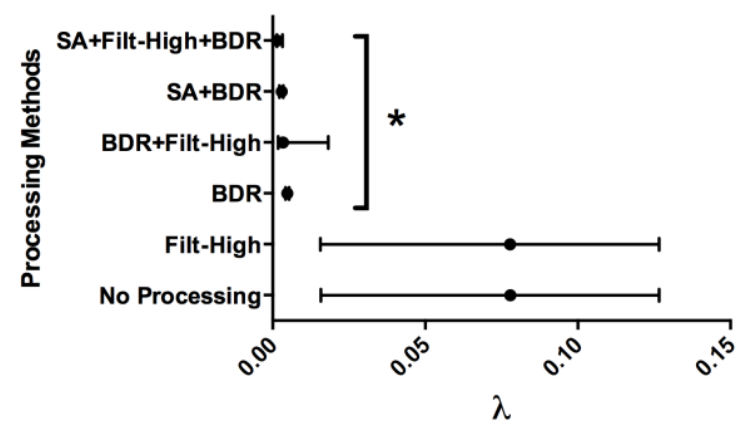

Figure 7. Regularization parameters $(\lambda)$ computed by the L-curve method using torso signals processed with the different processing methods.

\section{Discussion}

In this preliminary study we have evaluated the impact of different signal processing methods on a standard formulation of the inverse problem using experimental model. Processing methods, categorized into three distinct groups (high frequency noise removal, baseline drift removal and signal averaging), had variable impacts on the inverse problem reconstructions.

The major findings of this stidy are that the removal of high-frequency noise (through Filt-High or SA) from the torso potentials resulted in smoother reconstructed electrograms, facilitating the computation of activation times as high-frequency noise can make marker placement ambiguous.

Torso signals without BDR (i.e. no-processing or FiltHigh) resulted in a higher regularization parameter choice using the L-curve method (Figure 2). The more baseline drift present in these signals, the more regularization the signals received, resulting in very low amplitude signals as measured by RMS voltage, but also smoother reconstructed signals. Conversely, reconstructions after BDR improved the amplitude of electrograms but with it the amplitude of high-frequency noise. This noise was too great in most cases and resulted in substantial inaccuracies during activation time derivations (Figure 6).

Neither Filt-High nor BDR methods substantially altered the global topology of reconstructed electrograms (Figure 4). However, SA did change the topology. In very small regions of the heart, it was found that reconstructed electrograms after SA were different to other inverse solutions, with the intrinsic deflection no longer aligned with those recorded. Despite this, activation times were still accurately derived when using a global fitting approach [8]. We believe this morphology change was due to slight inaccuracies in the alignment of the QRS during averaging, resulting in error being introduced into the signals and thus inverse solutions.

Further investigation into the impact of these processing methods on inverse reconstruction is underway using additional data sets and different inverse methods.

\section{Conclusions}

The signal processing methods used have a dramatic effect on inverse problem reconstruction accuracy, particularly on the magnitude of electrograms and the activation time derived from these signals.

\section{Acknowledgements}

For the Consortium on ECG Imaging (CEI), all authors contributed equally. This work was supported by the French National Research Agency (ANR-10-IAHU04LIRYC), La Fondation Coeur et Artères (FCA14T2), the European Research Council under the European Union's Seventh Framework Programme (FP/2007-2013), the Fondation Leducq Transatlantic Network of Excellence RHYTHM network (16CVD02) and VEGA 2/0071/16 and APVV-14-0875 in Slovakia.

\section{References}

[1] Dubois R, Shah AJ, Hocini M, Denis A, Derval N, Cochet H, Sacher F, Bear L, Duchateau J, Jais P, Haissaguerre M. Non-invasive cardiac mapping in clinical practice: Application to the ablation of cardiac arrhythmias. Journal of Electrocardiology. 2015;48(6):966-74.

[2] Bear LR, LeGrice IJ, Sands GB, Lever NA, Loiselle DS, Paterson DJ, Cheng LK, Smaill BH. How Accurate Is Inverse Electrocardiographic Mapping?: A Systematic In Vivo Evaluation. Circulation: Arrhythmia and Electrophysiology. 2018;11(5):e006108.

[3] Ramanathan C, Rudy Y. Electrocardiographic imaging: II. Effect of torso inhomogeneities on noninvasive reconstruction of epicardial potentials, electrograms, and isochrones. J. Cardiac Electrophysiology. 2001:12(2) 24152.

[4] Bear LR, Huntjens PR, Walton RD, Bernus O, Coronel R, Dubois R. Cardiac electrical dyssynchrony is accurately detected by noninvasive electrocardiographic imaging. Heart Rhythm. 2018;15(7):1058-69.

[5] Svehlikova J, Zelinka J, Dogrusoz, Y, Good, W, Tysler M, Bear, L. Impact of Signal Preprocessing on the Inverse Localization of the Origin of Ventricular Tachycardia. CINC 2018 (In press).

[6] Tikonov AN, Arsenin VY. Solutions of ill-posed problems. New York: Winston. 1977.

[7] Hansen PC, O'Leary DP. The use of the L-curve in the regularization of discrete ill-posed problems. SIAM Journal on Scientific Computing. 1993;14(6):1487-503.

[8] Duchateau J, Potse M, Dubois R. Spatially coherent activation maps for electrocardiographic imaging. IEEE Trans. Biomed. Engineering. 2017;64(5):1149-56.

Dr. Laura Bear.

IHU-Liryc, Site du Hôpital Xavier Arnozan, Avenue du Haut Lévèque, 33600 Pessac, France. laura.bear@ihu-liryc.fr 